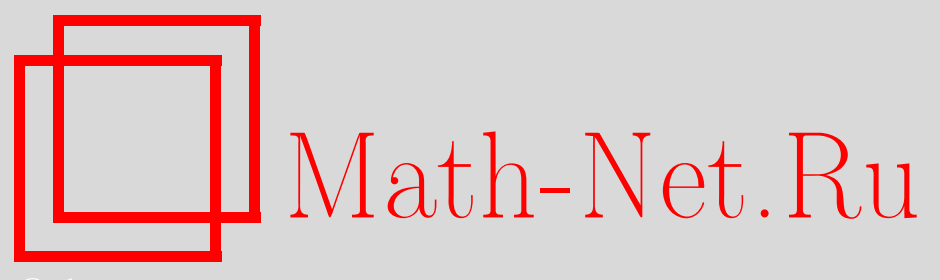

Л. В. Жижиашвили, О точечной расходимости общих тригонометрических рядов, Матем. заметки, 2002, том 71, выпуск 2, 315-317

DOI: https://doi.org/10.4213/mzm643

Использование Общероссийского математического портала Math-Net.Ru подразумевает, что вы прочитали и согласны с пользовательским соглашением http://www. mathnet.ru/rus/agreement

Параметры загрузки:

IP : 54.196 .121 .252

26 апреля 2023 г., 15:40:33

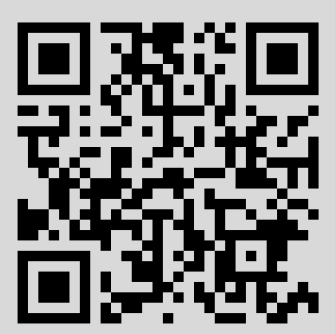




\section{О ТОЧЕЧНОЙ РАСХОДИМОСТИ ОБЩИХ ТРИГОНОМЕТРИЧЕСКИХ РЯДОВ}

\section{Л. В. Жижиашвили}

Настоящая работа посвящается восьмидесятилетию со дня рождения известного русского математика Сергея Борисовича Стечкина (1920-1995).

Пусть

$$
\frac{a_{0}}{2}+\sum_{k=1}^{\infty} a_{k} \cos k x+b_{k} \sin k x
$$

- тригонометрический ряд, а

$$
\frac{a_{0}}{2}+\sum_{k=1}^{\infty} b_{k} \cos k x-a_{k} \sin k x
$$

- ему сопряженный. Формально ряды (1) и (2) являются соответственно вещественной и мнимой частями степенного ряда

$$
\frac{a_{0}}{2}+\sum_{k=1}^{\infty} c_{k} z^{k}
$$

где $c_{k}=a_{k}-i b_{k}, z=e^{i x}, x \in[-\pi, \pi]$.

В 1906 году Фату [1] поставил вопрос: существует ли расходящийся на множестве положительной лебеговой меры тригонометрический ряд с коэффициентами, стремящимися к нулю?

В 1911 году Лузин [2] решил задачу Фату в более сильной форме: существует степенной ряд (3) с коэффициентами, стремящимися к нулю, расходящийся в каждой точке единичной окружности $\Gamma=\{z:|z|=1\}$. Лузин доказал, что для построенного степенного ряда оба ряда (1) и (2) расходятся почти всюду. В 1957 году Стечкин [3] показал, что на самом деле вещественная и мнимая части степенного ряда, построенного Лузиньм, расходятся всюду.

В 1912 году Штейнгауз [4] построил более простой тригонометрический ряд

$$
\sum_{k=4}^{\infty} \frac{\cos k(x-\ln \ln k)}{\ln k}
$$

которьй расходится всюду.

В 1959 году О'Шеа [5] рассматривал тригонометрические ряды вида

$$
\sum_{k=1}^{\infty} u_{k} \cos k q(k) \cos k x
$$

с $u_{k} \downarrow 0$ и показал, что при определенных условиях, наложенных на последовательности $\left(u_{k}\right)_{k} \geqslant 1$ и $(q(k))_{k \geqslant 1}$, ряд (5) расходится всюду.

Именно, имеет место 
УТВЕРЖДЕНИЕ. Пусть последовательности

$$
u(n) \uparrow \infty, \quad c_{n} \downarrow 0, \quad n \rightarrow \infty,
$$

и пусть существует последовательность чельх положительных чисел $\left\{p_{n}\right\}$ такал, что

$$
\begin{gathered}
\limsup _{n \rightarrow \infty}\left(n+p_{n}\right)\left(u\left(n+p_{n}\right)-u(n)\right)<\frac{1}{2}, \\
\liminf _{n \rightarrow \infty} \sum_{n+1}^{n+p_{n}} c_{r}>0 .
\end{gathered}
$$

Тогда последовательность

$$
\sum_{n=1}^{\infty} c_{n} \cos n u(n) \cos n x
$$

расходится для всех натуральных $x$, а последовательность

$$
\sum_{n=1}^{\infty} c_{n} \sin n u(n) \sin n x
$$

расходится $\partial$ яя всех $x \not \equiv 0(\bmod \pi)$.

Условия этого утверждения, в частности, вьполнены для "косинус-части" ряда (4). Из результатов О'Шеа [5] следует также основное утверждение работы Херцога [6].

В семидесятые годы Стечкин читал лекции в Тбилисском государственном университете. В личных беседах он ставил следующие вопросы.

ЗАдАчА 1. Какие условия на последовательности действительных чисел $\left(u_{k}\right)_{k \geqslant 1}$ и $(q(k))_{k \geqslant 1}$ обеспечивают расходимость всюду ряда

$$
\sum_{k=1}^{\infty} u_{k} \cos k(x-q(k))
$$

если предполагать, что $u_{k} \downarrow 0$ при $k \uparrow \infty$ ?

ЗАДАчА 2. Можно ли усилить результаты О'Шеа [5] и распространить их на тригонометрические ряды вида

$$
\begin{aligned}
& \sum_{k=1}^{\infty} u_{k} \cos k q(k) \sin k x, \\
& \sum_{k=1}^{\infty} u_{k} \sin k q(k) \cos k x, \\
& \sum_{k=1}^{\infty} u_{k} \sin k q(k) \sin k x ?
\end{aligned}
$$

В настоящей работе излагаются полученные нами утверждения, дающие ответ на эти вопросы Стечкина.

Справедливы следующие утверждения. 
ТЕОРЕМА 1. Пусть $\left(u_{k}\right)_{k \geqslant 1}$ - монотонно убивающая $\kappa 0$ последовательность вещественных чисел, причем

$$
\sum_{k=4}^{\infty} \frac{u_{k}}{k}=+\infty
$$

$a(p(n))_{n \geqslant 1}-$ возрастающая последовательность натуральных чисел такая, что

$$
\liminf _{n \rightarrow \infty} \sum_{k=n+1}^{n+p(n)} u_{k}>0
$$

Тогда если монотонно возрастающая $\kappa$ бесконечности последовательность полохительных вещественных чисел $(q(n))_{n \geqslant 1}$ удовлетворяет условию

$$
\limsup _{n \rightarrow \infty}[n+p(n)] \cdot[q(n+p(n))-q(n)]<\frac{\pi}{2},
$$

то тригонометрический ряд (6) расходится всюду.

ТЕОРема 2. Если монотонные последовательности $\left(u_{k}\right)_{k \geqslant 1} u(p(n))_{n \geqslant 1}$ удовлетворяют условиям (10) и (11), а для монотонно возрастающей к бесконечности последовательности полохительных вещественных чисел $(q(n))_{n \geqslant 1}$ имеем

$$
\limsup _{n \rightarrow \infty}[n+p(n)] \cdot[q(n+p(n))-q(n)]<2 \arcsin \frac{1}{4},
$$

то ряд (5) расходится всюду, а ряд (7) расходится во всех точках $x \neq 0(\bmod \pi)$.

ТЕОРема 3. Если монотонные последовательности $\left(u_{k}\right)_{k} \geqslant 1 u(p(n))_{n \geqslant 1}$ удовлетворяют условиям (10) и (11), а для монотонно возрастающей к бесконечности последовательности положительных вещественных чисел $(q(n))_{n \geqslant 1}$ имеем

$$
\limsup _{n \rightarrow \infty}[n+p(n)] \cdot[q(n+p(n))-q(n)]<2 \arcsin \frac{1}{16},
$$

то ряд (8) расходится всюду, а ряд (9) расходится во всех точках $x \neq 0(\bmod \pi)$.

Из сформулированных теорем можно получить различные следствия. Приведем наиболее характерное из них.

СледствиЕ. а) В условиях теоремы 1

$$
\sum_{k=1}^{\infty} u_{k}(2+\cos k q(k)) \cos k x
$$

есть всюду расходящийся ряд с положительными коэффициентами.

b) В условиях теоремы 2 ряд

$$
\sum_{k=1}^{\infty} u_{k}(2+\sin k q(k)) \cos k x
$$

есть всюду расходящийся ряд с положительными коэффициентами. 


\section{СПИСОК ЦИТИРОВАННОЙ ЛИТЕРАТУРЫ}

1. Fatou P. // Acta Math. 1906. V. 30. P. 335-400. 2. Luzin N. N. // Rendic. Circ. Mat. di Palermo. 1911. V. 32. P. 386-390. 3. Стечкин С. Б. // Изв. АН СССР. Сер. матем. 1957. Т. 21. № 5. C. 711-728. 4. Steinhaus H. // C. R. Soc. Sci. Varsovie. 1912. V. 5. № 3. P. 219-229. 5. Siobhan O’Shea. // Proc. Amer. Math. Soc. 1959. V. 10. № 1. P. 68-70. 6. Herzog E. // Michigan Math. J. 1953-1954. V. 2. № 2. P. 175-177.

Тбилисский государственный университет им. Ив. Джавахишвили 